\title{
Research on Behavior Prediction Based on Deep Learning - Take Chengdu Economic Innovation Enterprise as an Example
}

\author{
Jia Yuan ${ }^{1, *}$ \\ ${ }^{1}$ Chengdu Institute of Public Administration, Chengdu, China
}

\begin{abstract}
As the company's workforce continues to expand, finding key features related to employee performance, quickly identifying high-potential employees, and predicting a rise in turnover are hot spots for research. This paper first analyzes the key characteristics of dataset performance and applies deep learning to identify high-potential employees and predicts the rise of separation. Compared with traditional machine learning methods, it can be seen that deep learning applications have a greater improvement. The aim is to provide a new idea for the intersection of human resources and computer AI. In the preparation of this article, a large number of companies' desensitized employee data were collected in the real industry, including job, performance, education, and data communication between employees. Firstly, an interactive network-based employee topology map was established. According to the large amount of data collected from the real industry, the key characteristics of employee performance were analyzed, and a series of models were compared to traditional machine learning methods and deep learning calculation indicators, including accuracy, AUC and other indicators.
\end{abstract}

\section{Introduction}

According to a catalyst study, on average, the cost of replacing employees is about $50 \%$ to $75 \%$ of their annual salary. On average, it takes about 50 days to replace an employee, and the cost due to lost productivity will continue to increase. For a large company like Everi with more than 20000 employees, the total cost of annual turnover will rise to at least 270 million pesos, taking into account a $15 \%$ turnover rate and an average salary of 15000 pesos. Therefore, timely analysis of employee performance will help the company to better retain talents and save money. In company management, improving employee performance is a key issue, and understanding the main factors affecting employee performance is crucial for both managers and researchers [1-6].

There are many qualitative researches in the theoretical research and practical application of human resources discipline, which can not manage employees better through efficient methods. With the development of big data and computer, we can process tens of millions of data more conveniently, and explore the value of data through machine learning, data mining algorithm and big data technology. In the aspect of human resources, it is an urgent problem to quickly identify high potential employees and predict their promotion, resignation and performance through quantitative and scientific methods. At the same time, the effect of traditional machine learning methods is gradually surpassed by deep learning in various fields.
In order to realize the interdisciplinary research of computer science and human resource management, this study collects a large number of company data, collects personnel management data and employee network communication data, uses big data technology to process data and calculate key performance characteristics, and uses machine learning and deep learning technology.

\section{Performance analysis}

Talent is the most important existence of enterprises, and performance management is to give full play to the initiative of talents. Once the performance indicators are determined, employees will try to find various ways to strive for the available resources to complete and achieve all the performance goals, and all employees can focus on a common direction and goal. Through the results of performance, enterprises can understand the working status of employees at that time, so as to better manage employees. For employees, through the assessment to find problems, improve the existing problems, find the gap to improve, through the performance assessment, the recruitment of employees, promotion and demotion, internal learning, wage levels are combined, so that the incentive role of the enterprise can be given full play, and also contribute to the healthy development of the enterprise; in this process, it is also convenient for the employees to establish a continuous self driven behavior mode, which is a win-win result. In fact, the human resources department has always wanted to fully understand the work status of employees, but due to the different types of work, there is little intersection

*Corresponding author: joyce82513@163.com 
between the human resources department and employees. However, due to the development of the Internet, employees often work online, which inadvertently leaves a lot of information, such as communication information and sending e-mail information. This information avoids the deviation caused by the form of questionnaire or active inquiry, because employees will have a sense of psychological preparedness, which will cause certain pressure on employees. Therefore, we establish a network diagram through the communication direction, frequency and other information between employees, and calculate the Pearson correlation coefficient (PCC) of performance according to the calculated characteristics to analyze the key characteristics of employee performance. PCC represents the correlation between the two variables, and the larger the value is, the more relevant it is.

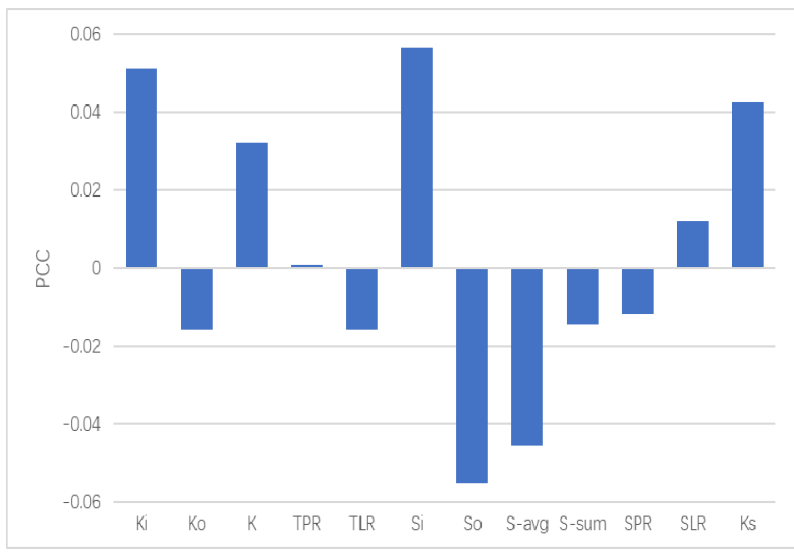

Fig. 1. Correlation coefficient chart of performance score and each index in data set 1.

Calculate data set 1, which contains the data of sending and receiving official documents among employees to form a network diagram. As can be seen from Figure 1, half of the characteristics are positively correlated with performance, namely, degree, PageRank index of powerless graph, entry intensity, leader rank index with weight, and core index. The more obvious positive correlation is degree, which represents that this kind of employees have to deal with many other employees' affairs, and that they have greater responsibility in the enterprise, Degree is the sum of out degree and in degree, which means that the more people communicate with others, the more active they are, and the easier they are to get high performance. In addition, there is the intensity of input, which represents the frequency of other people's communication with him. The higher the frequency, the more likely this person is to be responsible for, and the easier it is to get higher performance. Finally, the core indicators of the network represent that the more central position in the employee network diagram, the easier it is to get higher performance. For the indicators that are significantly negatively correlated, there are degree and intensity. These two indicators indicate that the more you ask others about communication, the lower performance employees may be, because most of the employees at the lower level communicate more with the employees at the higher level.

The employee communication network diagram is shown in Figure 2 below.

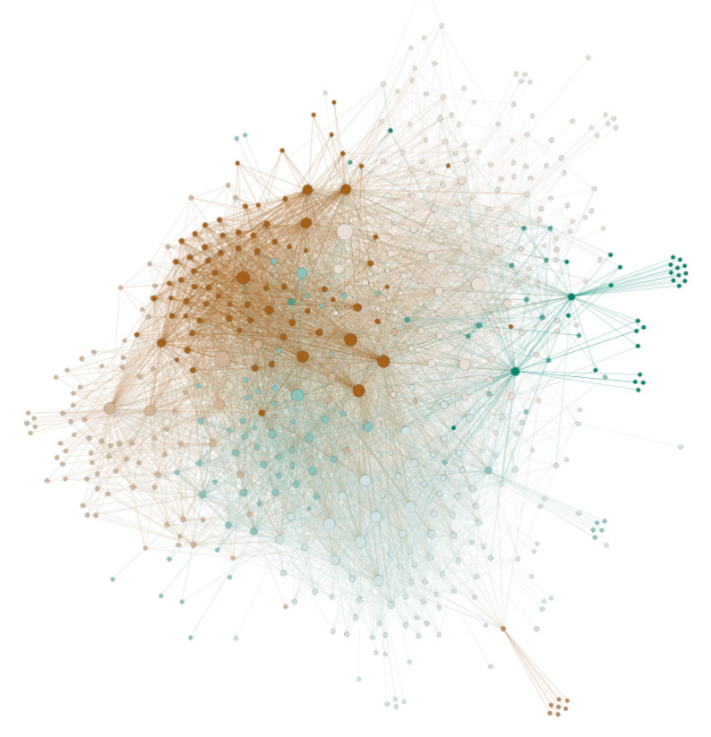

Fig. 2. Communication network diagram

\section{Promotion forecast}

In order to improve the enthusiasm of each enterprise member, many enterprises have implemented various promotion mechanisms to provide a fair and just competition environment for employees. Only when the ability of employees is affirmed can they create greater value for the enterprise, and everyone can do their best to contribute to the enterprise. The transfer of employee positions will cause the adjustment of employee relationship and the change of internal mechanism, so a good prediction system of employee promotion mechanism is particularly important. With the growing scale of enterprises and the development of big data technology, it can better predict the promotion of employees in advance, so as to provide excellent candidates for enterprises, which is particularly important when the company becomes larger. And sometimes some characteristics of employees, such as poor communication, can provide more objective data to the company through the information left by employees subconsciously. By means of big data analysis, we can better quantify all aspects of employees. By establishing the promotion model of employees, we can automatically find high-quality promotion candidates for enterprises. Through the factor analysis method of this study, enterprises can establish their own talent promotion mechanism, carry out differentiated management on employees, and make the results interpretable and automatic.

Based on the collected data, this study analyzes the factors of employee promotion, and predicts the promotion behavior according to the existing characteristics. Similar to the modeling method in the 
previous section, this section continues to analyze the differences between traditional machine learning methods and deep learning through the promotion prediction module. On the label, we choose the promoted employee as 1 and the others as 0 .

\subsection{Promotion prediction based on traditional machine learning}

Because the number of promotion and resignation is relatively small, the use of tree lifting algorithm is easy to cause over fitting, so here we choose the logistic regression algorithm. It is a very common model in machine learning, and it is often used in the actual production environment. Logistic regression is a classification algorithm, which is to distinguish the promoted employees from other employees.

Using the algorithm and cross validation, the average accuracy of data 8 is 0.8906 . The average accuracy of data 10 is 0.79 .

\subsection{Using deep learning to predict promotion}

The network structure is used to calculate the collected features. After fine tuning the parameters, the accuracy of the verification set in data set 6 is 0.9333 . The accuracy on the validation set of data set 7 is 0.8132 . It is superior to the traditional machine learning methods in performance.

\section{Turnover forecast}

With the change and development of the times, the emergence of computer network and the realization of resource sharing, the competition between enterprises is no longer limited to resources, equipment and ideas, but more talent competition. The role of talents is the basis of enterprise development, and the loss of talents is crucial to the rise and fall of enterprises. Each department of the company has a group of professionals, they can improve the operation efficiency of each department of the enterprise, but on the other hand, if the enterprise lacks such talents, it will not only lead to low efficiency, but also a serious waste of various resources of the enterprise. In addition, the brain drain is not conducive to the innovation and sales of enterprise products, so that the enterprise can not develop stably for a long time. If employees find that their working hours, wages and benefits and work content are different from what they know, they may feel cheated and even want to resign. Managers who are rude or lack of communication with employees often make employees think about leaving. On the contrary, if managers can spend some time communicating with new employees and meeting with them regularly, they can create a good working environment. The adaptability, knowledge and social skills of high potential employees should be the best. However, if these people are not found and cultivated by the leaders, then these excellent high potential employees are likely to leave, or some people with low ability are arranged in an inappropriate position, lacking certain communication skills, teamwork skills and organizational leadership skills, Then these employees will be forced to leave by various pressures. If the enterprise can not accurately predict which employees will leave in the future, it will face the vacancy of enterprise posts, brain drain and low work efficiency. This will not only lead to the reduction of enterprise productivity, but also may lead to the collapse of enterprise production chain. Therefore, it is very important to predict the future turnover of employees. If the enterprise can accurately predict the future turnover of employees, it can avoid the risk of employee turnover in advance, supplement manpower in time, and make the enterprise production run normally. The problem of employee turnover is also a problem that every enterprise has to face, especially the turnover of excellent talents is a major loss of enterprises.

After cross validation, the average accuracy of 0.9714 is obtained on data 8 . The average accuracy of 0.8025 is obtained on data 9 .

The network structure is used to calculate the collected features, and after fine-tuning the parameters, the accuracy rate on the verification set of data set 8 is 0.9688 . The accuracy is 0.7800 on dataset 9 . It is slightly lower than the traditional machine learning method.

This section first demonstrates the advantages and disadvantages of traditional machine learning method and deep learning method on the task of identifying high potential employees. The machine learning method adopts xgboost lifting tree model, briefly describes the principle and mathematical expression of the algorithm, and deep learning uses the network structure to predict. Then the task experiment results show that the results of the two methods are low, which shows that there are other aspects of the potential of high potential employees, which need to be further studied. In addition, deep learning is better than traditional machine learning in the result. Secondly, in the promotion prediction task, we first analyzed the correlation coefficient between performance and education background, age, length of service, etc. in this data set, we found that the key factors related to performance were positively correlated with age and length of service, and negatively correlated with education background. It also analyzes the relationship between education background and age, because of the proportion of old employees and the difficulty of obtaining education background. It also analyzes the correlation coefficient between education background, length of service, age, performance and promotion, and concludes that promotion is positively correlated with performance, education background and age, and negatively correlated with length of service, which is caused by the limited promotion space of senior employees due to their higher level. In addition, the comparison between machine learning method and deep learning method of promotion prediction is also made, as shown in Table 1. The machine learning adopts the logistic regression algorithm, and the average accuracy of 0.8906 is obtained by cross validation. The deep learning method continues to use the network structure, and fine tune the parameters, and finally obtains the 
accuracy of 0.9333 , which is $4.7 \%$ higher than the traditional machine learning method.

Finally, in terms of turnover prediction, this paper first analyzes the correlation between education background, age, length of service, performance and turnover, and finds that turnover is directly proportional to education background and performance, which indicates that the higher education background and performance, the more willing to quit, and to a certain extent, the more ability, the easier job hopping; On the contrary, it was negatively correlated with age and length of service. Because the older the age and the longer the length of service, the more the pursuit of stability and technical limitations, and the weaker the competitiveness in the talent market, the easier it is to stay in the company. However, the prediction accuracy of data set 9 is very low, which indicates that the company's turnover has a certain randomness.

Table 1. Performance comparison of traditional machine learning algorithm and deep learning algorithm on promotion and turnover prediction accuracy.

\begin{tabular}{|c|c|c|}
\hline & $\begin{array}{c}\text { Traditional } \\
\text { machine learning }\end{array}$ & Deep learning \\
\hline $\begin{array}{c}\text { Data 8 promotion } \\
\text { forecast }\end{array}$ & 0.8906 & $\mathbf{0 . 9 3 3 3}$ \\
\hline $\begin{array}{c}\text { Data 9 promotion } \\
\text { forecast }\end{array}$ & 0.7600 & $\mathbf{0 . 8 1 3 2}$ \\
\hline $\begin{array}{c}\text { Data 8 turnover } \\
\text { forecast }\end{array}$ & $\mathbf{0 . 9 7 1 4}$ & 0.9688 \\
\hline $\begin{array}{c}\text { Data 9 turnover } \\
\text { forecast }\end{array}$ & $\mathbf{0 . 8 0 2 5}$ & 0.7800 \\
\hline
\end{tabular}

\section{Conclusion}

The work of this study first collected a large number of company personnel data and network communication data, and then through the construction of interactive network, constructed three kinds of network diagram: directed powerless network diagram, directed weighted network diagram, undirected weighted network diagram, obtained 12 kinds of network characteristics through graph network calculation, through these characteristics for performance analysis. Then, it becomes the input feature to identify high potential employees, and tests the accuracy of traditional machine learning method and deep learning method. Then, through other data sets, we get the information of educational background, promotion and turnover, and analyze the correlation between other characteristics and performance. Then, in order to predict promotion and turnover, we use traditional machine learning method and deep learning method to compare. The experimental results show that deep learning is better than machine learning in high potential employee identification and promotion prediction; It is slightly lower than the traditional machine learning method in turnover prediction. It can be seen that in the identification of high potential employees, the accuracy of identification is not high, mainly because the characteristics of high potential employees need to be explored. It is hoped that this research can promote other researchers to continue to pay attention to the research of deep learning in human resource management, and further in-depth research in this cross field in the future.

Employees are very valuable wealth for enterprises. Enterprises should use retention analysis to protect the most valuable assets of enterprises. In the global competitive world, talent is the most important issue for most CEOs. Executives worry about how to acquire and retain the best and brightest talents, and how to cultivate, maintain and develop their star employees. The demand of enterprises for highly skilled personnel continues to grow, and the competition for talent reserve is becoming increasingly fierce. Another challenge facing enterprises is that employees pursue healthy and good environment, value goals and community, and hope to achieve a balance between work and life. The talent problem is more complicated than ever. The retention and loss of talents is the biggest challenge among all the problems. How to retain key talents and high potential talents is the most concerned thing for most business leaders and human resource leaders. More and more mature companies have begun to use advanced analysis methods and big data to help them control employee retention problems, so that these problems can be quantified and solved more quickly and flexibly. These companies do not use data analysis to help them find a glimmer of hope, but use data analysis as a tool to constantly adjust their understanding of the retention problem, and put forward flexible and novel solutions in real time to reduce the retention problem. From machine learning technology to construction algorithm, the continuous learning behavior using big data integrates as many dimensions as possible, making these types of talent retention solutions more advanced and quantifiable.

\section{References}

1. Cai M, Du H, Zhao C, et al. Relationship between employees' performance and social network structure: An empirical research based on a SME from a whole-network perspective[J]. Chinese Management Studies, 2014, 8(1): 85-108.

2. Gaggioli A, Mazzoni E, Milani L, et al. The creative link: Investigating the relationship between social network indices, creative performance and flow in blended teams $[\mathrm{J}]$. Computers in Human Behavior, 2015, 42: 157-166.

3. Lee H, Werner S, Kim T Y. High performance work systems and organization attraction: The moderating effects of vocational interests[J]. Employee Relations, 2016, 38(5): 682-702.

4. Paruchuri S, Awate S. Organizational knowledge networks and local search: The role of intraorganizational inventor networks[J]. Strategic Management Journal, 2017, 38(3): 657-675.

5. Singh H, Kryscynski D, Li X, et al. Pipes, pools, and filters: How collaboration networks affect innovative performance[J]. Strategic Management Journal, 2016, 37(8): 1649-1666.

6. Xie Y. It's whom you know that counts[J]. Science, 2017, 355(6329): 1022-1023. 